\title{
Asma: uso adecuado de dispositivos para inhalación
}

\author{
José R. Fernández-Soto ${ }^{1}$, Elsy M. Navarrete-Rodríguez ${ }^{1 *}$, Blanca E. Del-Río-Navarro ${ }^{1}$, \\ Omar J. Saucedo-Ramírez', Jaime M. Del-Río-Chivardi', Néstor A. Meneses-Sánchez', \\ Laura Gochicoa-Rangel ${ }^{2}$ y Juan J. Sienra-Monge ${ }^{3}$
}

${ }^{1}$ Servicio de Alergia e Inmunología, Hospital Infantil de México Federico Gómez; ${ }^{2}$ Departamento de Fisiología Respiratoria, Instituto Nacional de

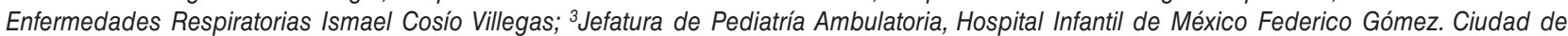
México, México

\begin{abstract}
Resumen
La terapia inhalada se considera la piedra angular del manejo del asma. Sin embargo, a pesar de ser la forma ideal de administración de estos medicamentos, solamente el $70 \%$ de los pacientes cumple el tratamiento adecuadamente y sólo del 39 al $67 \%$ de los médicos conocen y pueden explicar de forma adecuada las distintas técnicas de inhalación. La terapia inhalada tiene características muy particulares. El depósito pulmonar de un medicamento inhalado a través del tracto respiratorio es más complejo que cuando se administra por vía oral, y varía dependiendo de varios factores, tanto inherentes al medicamento como a la forma de administrarlo. Para que la terapia inhalada sea exitosa, se requiere que se generen partículas del medicamento de un tamaño apropiado que penetren más allá de la orofaringe y la laringe, y que puedan depositarse en los pulmones. Existen múltiples dispositivos para la administración de medicamentos en la vía respiratoria baja. Cada uno ha probado tener una eficacia similar, siempre y cuando se utilicen con la técnica correcta. La decisión para su uso se realiza con base en la edad del paciente, la capacidad de coordinar entre la inhalación y la activación del dispositivo y la presencia de síntomas agudos. La elección del dispositivo a utilizar siempre deberá hacerse de forma conjunta, evaluando pros y contras de cada uno de los dispositivos y siempre de forma individualizada.
\end{abstract}

Palabras clave: Asma. Nebulizadores. Vaporizadores. Inhalaciones. Tratamiento.

\section{Asthma: correct use of inhalation drug-delivery devices}

\section{Abstract}

Inhaled therapy is considered the cornerstone of asthma treatment. However, despite being the ideal form of drug delivery, it is recognized that only $70 \%$ of patients have an adequate attachment to their treatment and only $39-67 \%$ of physicians can explain the optimal inhaler technique. Inhaled therapy has very specific characteristics. Pulmonary deposit of an inhaled medication through the respiratory tract is more complex than when administered orally and depends on several factors inherent to both the medication and the administration. For successful inhaled therapy, the drug needs to be converted into particles of an appropriate size, which can enter beyond the oropharynx and larynx, and be deposited in the lungs. There are multiple devices for the administration of drugs in the lower respiratory tract, each one with a similar efficacy as long as

Correspondencia:

*Elsy Maureen Navarrete-Rodríguez E-mail: draenavarrete@gmail.com
Disponible en internet: 11-01-2019 Bol Med Hosp Infant Mex. 2019;76:5-17

www.bmhim.com

1665-1146/@ 2019. Hospital Infantil de México Federico Gómez, impreso por Permanyer México SA de CV, todos los derechos reservados. 
it is used with the correct technique. The decision of which device should be used is made based on the age of the patient, the ability to coordinate between the inhalation and activation of the device, and the presence of acute symptoms. The choice of the device must be evaluated individually.

Key words: Asthma. Nebulizers. Vaporizers. Inhalation. Treatment.

\section{Introducción}

El asma constituye un grave problema de salud a escala mundial. Actualmente afecta a más de $300 \mathrm{mi}-$ llones de personas ${ }^{1}$ y tiene una prevalencia estimada del 1 al 18\%2. Su frecuencia se ha visto incrementada en los últimos años, y dada la importancia que tiene el padecimiento y la derrama económica que supone su mal manejo, se han realizado muchos esfuerzos para mejorar el tratamiento de los pacientes que la presentan.

Es bien sabido que la piedra angular del manejo del asma es la terapia inhalada ${ }^{3}$. Sin embargo, a pesar de esto, se reconoce que solamente el $70 \%$ de los pacientes cumple el tratamiento adecuadamente ${ }^{4}$, y sólo del 39 al $67 \%$ de los médicos conocen y pueden explicar las técnicas de inhalación adecuadas ${ }^{5}$.

La primera referencia conocida de la administración de un aerosol con fines terapéuticos es el papiro de Ebers (1554 a.C.), en el que se describía la inhalación del vapor que desprendían las plantas de beleño negro (Hyoscyamus niger) en pacientes con dificultad para respirar 6 . No fue hasta 1858 que se presentó, en la Academia de Medicina de París, el primer inhalador «a presión", inventado por Sales Girons en Francia. Seis años después, en Londres, Newton patentaba un aparato de inhalación para la administración de medicamentos en polvo seco ${ }^{7}$. Para la primavera de 1955, la hija del director general de Riker Laboratories, Inc., le preguntó a su padre por qué no podía tener su medicamento para el asma en una lata de aerosol como el espray de cabello ${ }^{8}$. Para ese momento, los pacientes con asma se administraban su medicamento broncodilatador a través de un nebulizador de mano sin la posibilidad de medir una dosis reproducible ${ }^{9}$, lo que provocaba gran variación en los efectos terapéuticos. En el verano de ese año se iniciaron los primeros ensayos clínicos con un prototipo de inhalador de dosis medida presurizada (pMDI), con lo que se demostró su eficacia. Los primeros pMDI, sulfato de isoproterenol y bitartrato de epinefrina, fueron lanzados en marzo de $1956^{\circ}$. En la década subsecuente, se inició el desarrollo de espaciadores y cámaras con válvula de retención, lo que evidencia que, para alcanzar el control de los síntomas del asma, no solo importaba el tipo de fármaco, sino también el dispositivo y la técnica utilizada para su administración?

Cuando se habla de terapia inhalada, se debe tomar en cuenta que esta tiene características muy particulares. El depósito pulmonar de un medicamento inhalado a través del tracto respiratorio es más complejo que cuando se administra por vía oral y varía dependiendo de varios factores, tanto inherentes al sujeto como al medicamento y a la forma de administrarlo.

Dentro de los factores dependientes del sujeto, se debe considerar tanto la geometría del árbol bronquial como el patrón ventilatorio y la mecánica pulmonar. Ambos factores se describen a continuación:

- Geometría del árbol bronquial. Las vías aéreas tienen aproximadamente 23 generaciones: las primeras 14 presentan músculo liso circunferencial y cartílago, y corresponden al $5 \%$ del total del árbol bronquial; las últimas siete abarcan el $95 \%$ de la superficie de la vía aérea y no presentan cartílago. El estiramiento y tracción son lo que permite a esta sección mantener su permeabilidad ${ }^{4}$. Debido a lo anterior, es de suponer que diferentes fármacos están dirigidos a diversas regiones. Por ejemplo, los $\beta 2$-agonistas generalmente están dirigidos a generaciones de vías aéreas más proximales que contienen músculo liso circunferencial, mientras que los esteroides presentan una distribución más uniforme, ya que sus receptores están presentes a lo largo de toda la vía aérea $^{10}$. A este hecho se debe sumar que existe un cambio en el diámetro circunferencial de la vía respiratoria conforme se avanza en cada generación, por lo que el tamaño de la partícula del fármaco administrado influirá directamente en el sitio de su depósito.

- Patrón ventilatorio y mecánica pulmonar. El grado de enfermedad pulmonar en el momento de la inhalación también influye significativamente en el depósito pulmonar: la inflamación, hipersecreción de moco y broncoconstricción reducen el diámetro de las vías respiratorias y aumentan la velocidad del flujo de aire y la turbulencia, lo que provoca el depósito del fármaco en las vías respiratorias proximales ${ }^{11}$. Otro dato importante a tomar en cuenta es que los niños tienen vías respiratorias más pequeñas que los adultos, y 
es probable que las vías respiratorias de pacientes asmáticos sean más estrechas que las vías respiratorias de sujetos sanos, debido a la inflamación, remodelación y broncoconstricción, característicos de este padecimiento ${ }^{12}$.

Con respecto a los factores inherentes al medicamento, dentro de los más importantes están el tamaño de la partícula, la velocidad de ingreso, el crecimiento higroscópico y la carga electrostática:

- Tamaño de la partícula. Para que la terapia inhalada sea exitosa, se requiere que se generen partículas de medicamento que penetren más allá de la orofaringe y la laringe y que se depositen en la vía aérea pequeña ${ }^{3}$. Dado que los aerosoles generan partículas de varios tamaños, al igual que los inhaladores de polvo seco $(\mathrm{DPI})^{1}$, se utiliza una medida "promedio", conocida como diámetro de la masa media aerodinámica (DMMA), cuyo significado es que el $50 \%$ de las partículas en el aerosol son más pequeñas y el $50 \%$ más grandes que la DMMA reportada ${ }^{4}$.

El diámetro aerodinámico se considera el factor relacionado con la partícula más importante que afecta el depósito del aerosol ${ }^{10}$. Las partículas $>5 \mu \mathrm{m}$ son más propensas a depositarse por impacto en la orofaringe y ser deglutidas ${ }^{13}$; las partículas de 4 a $5 \mu \mathrm{m}$ se depositan de manera primaria en las vías aéreas bronquiales/conductoras, y las partículas más pequeñas permanecen en la corriente de aire y son llevadas a las vías periféricas y la región alveolar (Fig. 1).

Al entrar en la vía aérea, las partículas se depositarán por tres mecanismos: impacto, sedimentación y difusión. Esto depende de la velocidad de inhalación y el tamaño de la partícula:

- Impacto. Se origina por el choque de las partículas de aerosol en la vía aérea y afecta generalmente a partículas $>5 \mu \mathrm{m}$ que se administran con alta velocidad de disparo o descarga y de flujo inspiratorio. Lo primero depende del dispositivo, y lo segundo, del paciente. Este mecanismo es muy importante para el depósito de fármacos en las primeras siete generaciones de bronquios ${ }^{4,13}, \mathrm{y}$ ocurre principalmente con agonistas $\beta 2$-adrenérgicos. Si el paciente se encuentra llorando, se debe evitar administrar el medicamento, ya que aumenta el impacto. Se recomienda realizar siempre inhalaciones lentas y estables en pacientes tranquilos, y no olvidar que se si se utiliza un inhalador de dosis medida, se debe de acompañar de una cámara espaciadora, y utilizar la técnica adecuada.

- Sedimentación. Es el asentamiento de partículas de entre $0.5-5 \mu \mathrm{m}$ por efecto de la gravedad ${ }^{13}$. Se

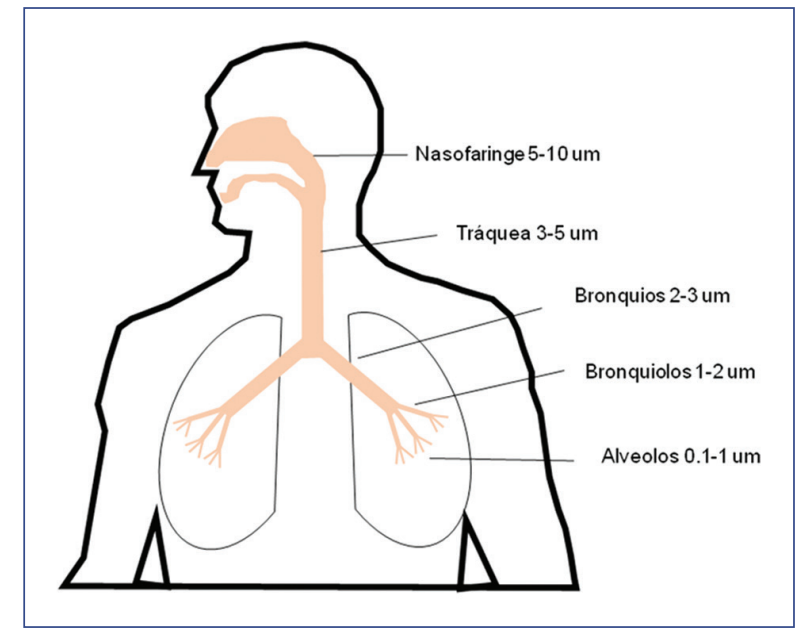

Figura 1. Tamaño de partículas inhalables en relación con el diámetro de la vía respiratoria.

produce predominantemente en vías aéreas de mediano calibre y más allá de la generación ocho, donde la velocidad de aire es menor ${ }^{4}$. La sedimentación se favorece durante una inhalación profunda y aplazando lo más que se pueda la exhalación para que las partículas se precipiten por gravedad y se pueda alcanzar un mayor depósito pulmonar.

- Difusión. Afecta a partículas muy pequeñas $k 1$ $\mu \mathrm{m})$ en regiones de bajo flujo pulmonar, donde el movimiento aleatorio de las mismas, denominado movimiento Browniano, tiene una mayor influencia que la gravedad ${ }^{14}$ (Fig. 2). Aunque las partículas pequeñas $(\approx 1 \mu \mathrm{m})$ tienen mayor potencial de ser exhaladas ( $10 \%$ de la dosis), la mayor parte de la dosis se deposita a nivel pulmonar (60\%), y una cantidad mucho menor a nivel orofaríngeo $(30 \%)^{15}$.

- Velocidad de ingreso. El flujo de aire influye directamente en el impacto de las partículas en las paredes de las vías aéreas y constituye uno de los aspectos más importantes en el depósito pulmonar. Una inhalación lenta y profunda después de una exhalación profunda, con una duración de 2 a $3 \mathrm{~s}$ en niños y de 4 a $5 \mathrm{~s}$ en adultos, genera flujos de $30 \mathrm{l} / \mathrm{min}$, que son ideales para pMDI y mínimos para DPI ${ }^{16}$. El flujo inspiratorio mayor a $60 \mathrm{l} / \mathrm{min}$ incrementa el impacto en la orofaringe y las vías respiratorias superiores, por lo que se debe de evitar hacerlo de manera precipitada. Con el uso de las cámaras espaciadoras se disminuye la velocidad de ingreso y el depósito orofaríngeo.

- Crecimiento higroscópico. La adición y eliminación de agua pueden afectar significativamente el tamaño de 


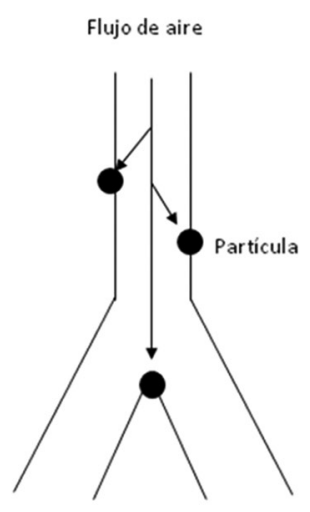

Impacto

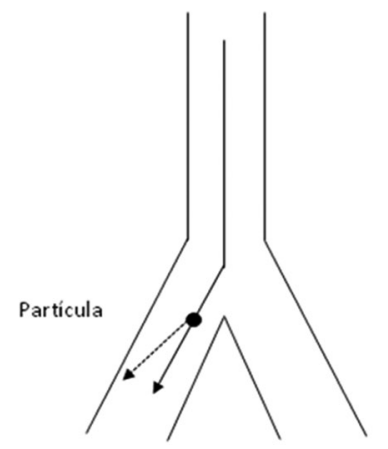

Sedimentación

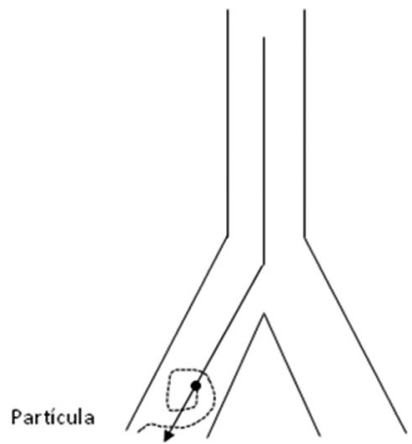

Difusión

Figura 2. Mecanismos de depósito pulmonar (modificado de Muchão, et al. ${ }^{14}$ ).

partícula de un aerosol higroscópico (que tiene tendencia a absorber humedad del ambiente) y la deposición. Algunas partículas pueden incrementar de tamaño en condiciones de alta humedad. Este crecimiento se encuentra en función del diámetro inicial de la partícula: si es $<1 \mathrm{~mm}$, puede incrementar hasta cinco veces su tamaño, mientras que si es $>2 \mathrm{~mm}$, el tamaño puede aumentar hasta tres veces. La humedad de la vía respiratoria es suficiente para generar estos cambios.

El aumento en el tamaño de partícula por encima del tamaño inicial afecta la cantidad de fármaco depositado y, particularmente, su distribución en la vía respiratoria ${ }^{13}$.

- Carga electrostática. La disponibilidad del medicamento en aerosol puede verse afectada por cargas electrostáticas presentes durante su generación o transporte, lo que afecta el depósito pulmonar ${ }^{17}$.

La carga electrostática de los aerosoles puede variar dependiendo de la formulación, el material utilizado en los dispositivos y las cámaras espaciadoras. Tratando de minimizar este problema, se han implementado diversas estrategias que tienen como objetivo mejorar la disponibilidad del medicamento en la vía respiratoria. Estas incluyen el uso de materiales antiestáticos en los dispositivos de inhalación o el cebar las paredes de la cámara espaciadora con 20 dosis del medicamento inhalado, para recubrir la superficie interna y minimizar la carga estática. Actualmente, la solución más práctica y menos costosa es usar espaciadores no conductores, lavarlos con detergente para trastes y secarlos al aire libre. Todo esto permite una mayor entrega final del medicamento ${ }^{18}$.

\section{Forma de administración: tipo de dispositivo}

El éxito de la terapia inhalada implica la adecuada elección del fármaco, la selección del dispositivo con el cual se administrará ${ }^{15}$ y, sobre todo, la correcta capacitación para su uso.

Un gran número de dispositivos de inhalación han sido diseñados en los últimos años. En general, se asume que el dispositivo ideal debería tener las siguientes características:

- Efectivo: Permite la inhalación de las partículas del medicamento $\leq 6 \mu \mathrm{m}$, independientemente del flujo inspiratorio del paciente.

- Reproducible: Permite la inhalación de la misma cantidad de medicamento siempre.

- Preciso: Permite saber el número de dosis del medicamento restante en el dispositivo.

- Estable: Es capaz de proteger el medicamento de los efectos de la temperatura y cambios de humedad.

- Cómodo: Es fácil de utilizar, fácilmente transportable, de tamaño pequeño, multidosis.

- Versátil: Permite el uso de diferentes combinaciones de medicamentos por inhalación.

- Amigable con el ambiente: No debe contener contaminantes químicos ni dañar la capa de ozono.

- Asequible: Tiene un precio justo y es recargable ${ }^{19}$.

En la actualidad, los dispositivos se agrupan dependiendo del tipo y forma de administración del medicamento:

- pMDI.

- DPI.

- Inhaladores de niebla fina (soft mist).

- Nebulizadores mecánicos y ultrasónicos. 
Todos han probado una eficacia similar, siempre y cuando se utilicen con la técnica correcta ${ }^{20}$. La decisión de su uso se toma basándose en la edad del paciente, la capacidad de coordinar entre la inhalación y la activación del dispositivo y en la presencia de síntomas de asma (esto afecta el flujo inspiratorio) ${ }^{21}$. Una vez considerados estos factores, y después de haber instruido al paciente en la técnica específica del dispositivo, el profesional de la salud debe solicitar una demostración para cerciorase de que se haga de manera correcta.

\section{Inhaladores de dosis medida presurizados}

Fue uno de los primeros dispositivos utilizados, cuya principal innovación fue la portabilidad. Antes de ellos se utilizaban aparatos de tamaño considerable para nebulizar el medicamento. Otra ventaja que supuso su uso fue la practicidad: con una técnica adecuada de inhalación profunda, se podía administrar la dosis del medicamento deseado solo y, finalmente, su característica de ser multidosis.

El pMDI es un dispositivo que genera un aerosol (sistema de dos fases definido como la dispersión de una suspensión de partículas sólidas o gotas líquidas en un medio gaseoso $)^{22}$ mediante el uso de un cartucho presurizado de aluminio que contiene el medicamento micronizado en una suspensión con etanol, como cosolvente, e hidrofluoroalcano (HFA), como propelente. Durante varias décadas se utilizó clorofluorocarbono (CFC) como propelente para los pMDI; sin embargo, este gas daña la capa de ozono. Como en 1987 se firmó el protocolo de Montreal, se estableció el cambio de CFC por HFA, el cual no afecta la capa de ozono ${ }^{23}$.

Los componentes estructurales del pMDI convencional son el frasco, válvula dosificadora, activador, pieza bucal, formulación y propelente ${ }^{24}$. La suspensión del medicamento se encuentra a una presión de 3 a 4 atmósferas y genera partículas de 2 a $4 \mu \mathrm{m}$. La válvula dosificadora está diseñada para entregar una cantidad precisa de aerosol (20-100 $\mu$ l) cada vez que el dispositivo se activa. Cuando el inhalador no se utiliza, una válvula interna entre el frasco y la cámara de medición se abre, lo que permite que la cámara se llene de una mezcla de propelente-medicamento en forma líquida. Al mismo tiempo, otra válvula externa entre la cámara de medición y el aire del exterior se cierra. Mientras el paciente presiona el frasco para la activación de la dosis, la válvula interna se cierra y la externa se abre, liberando la mezcla de medicamento-propelente, retenida en la cámara, a través del orificio del activador en forma de aerosol. Internamente, el activador incluye una boquilla de pulverización (orifico activador) y la cámara de expansión, en la cual el propelente liberado de la cámara de medición se expande y se volatiliza parcialmente debido a la disminución de la presión ${ }^{25-27}$ (Fig. 3).

A escala mundial, los pMDI son los dispositivos de inhalación más ampliamente utilizados para el tratamiento del asma y de la enfermedad pulmonar obstructiva crónica ${ }^{3}$ (Tabla 1).

\section{INHALADORES DE DOSIS MEDIDA PRESURIZADOS CON ESPACIADOR}

Un espaciador es un dispositivo de extensión que se sitúa entre el paciente y el $\mathrm{pMDI}^{28}$. Estos dispositivos ayudan a mejorar la eficiencia en el uso de los cartuchos presurizados al aumentar la distancia entre la boca y el dispositivo, lo que disminuye la velocidad de flujo del aerosol y el impacto en la orofaringe, y permite la evaporación del freón y la disminución del tamaño de la partícula. Además, facilitan la penetración y depósito pulmonar (mayor al 30\%).

Varios factores influyen sobre su eficacia, el espacio entre la entrada y la salida del inhalador, el material del que están hechos, la técnica de inhalación y el cuidado en general del dispositivo.

Existen diferentes tipos de espaciadores: el tubo abierto, el diseño de flujo inverso, en el cual el pMDI se sitúa cerca de la boca y es disparado en dirección lejana al paciente, y la cámara con reservorio o de retención, que es el más utilizado y que permite mantener el aerosol dentro de la cámara por un tiempo finito $^{3,29}$ (Fig. 4). Los hay de gran diversidad de tamaño: desde $50 \mathrm{ml}$ hasta $750 \mathrm{ml}$, lo cual puede impactar en la cantidad total del fármaco que se deposita en la orofaringe $o$ en los pulmones. Cuando el espaciador es pequeño se deposita menos medicamento a nivel pulmonar y más a nivel orofaríngeo. Inversamente, al aumentar la capacidad del espaciador, existe un mejor depósito a nivel pulmonar y menos a nivel orofaríngeo. Sin embargo, cuando se trata de pacientes con un volumen corriente pequeño (niños $\leq 2$ años), el volumen de espaciado grande requiere de tiempos más largos de administración ${ }^{30}$. Existen estudios donde los diferentes dispositivos espaciadores aumentan la biodisponibilidad del medicamento inhalado ${ }^{31,32}$. Sin embargo, el punto fundamental para la entrega del medicamento es una técnica adecuada ${ }^{33,34}$. 


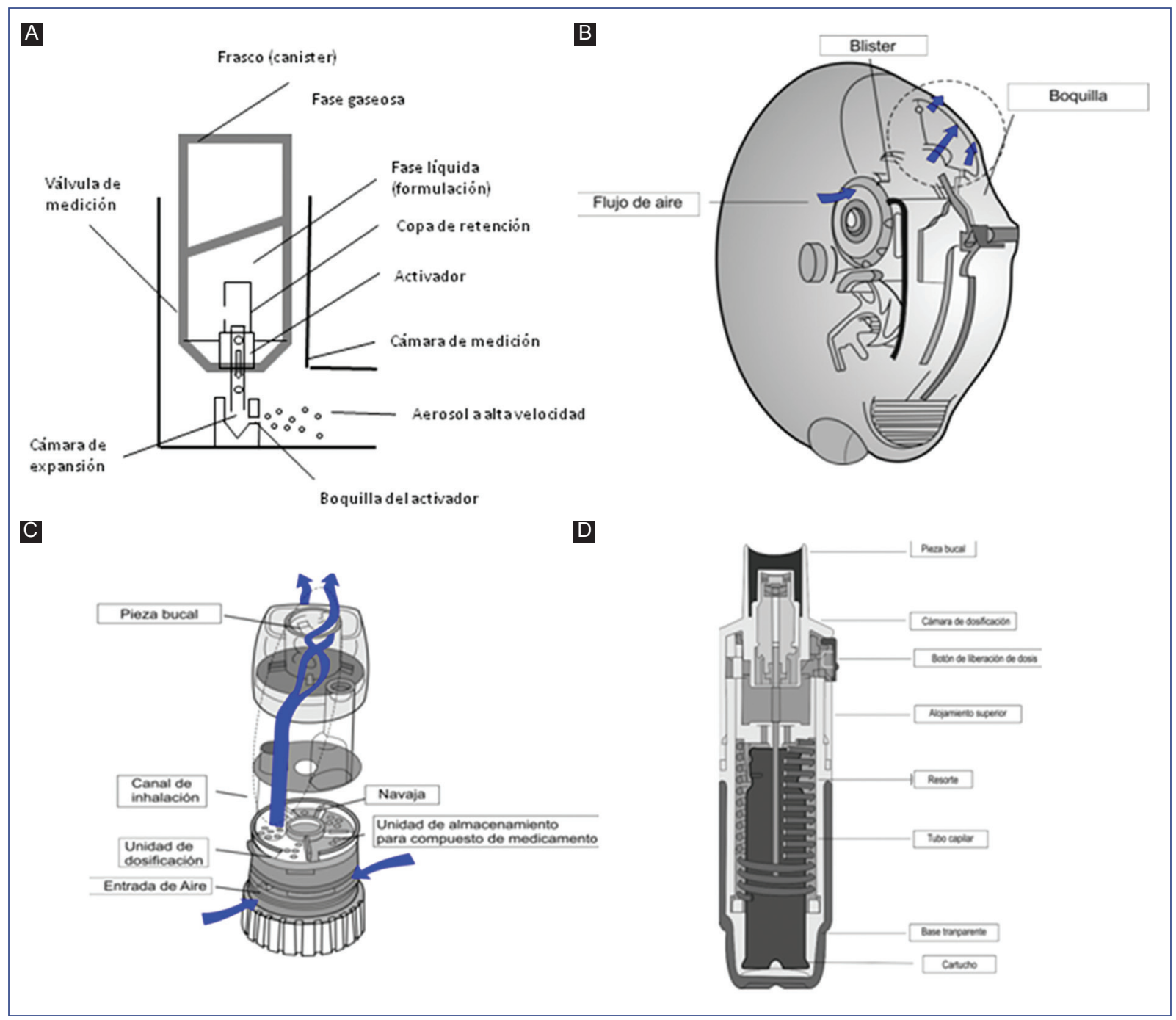

Figura 3. Componentes clave de los dispositivos: Inhaladores presurizados de dosis medida (A); polvo seco: Diskus ${ }^{\circledR}$ (B) y Turbuhaler $^{\circledR}$ (C); y Respimat ${ }^{\circledR}$ (D) (modificado de Newman SP y Azouz W, et al. ${ }^{26,27}$ ).

A pesar de las diferencias entre los diferentes espaciadores, todos tienen algunas ventajas inherentes a los diseños:

- Reducen la velocidad de las partículas del pMDI, lo cual se produce durante el tiempo que el aerosol atraviesa la longitud del espaciador o cámara de retención con válvula.

- Incrementan la fracción de partículas finas FPF debido al impacto de las partículas más grandes del medicamento y la evaporación parcial del propelente dentro de la cámara ${ }^{35}$.

- Superan, en parte, el problema de coordinar la activación con la inhalación, incrementando así el depósito pulmonar. No obstante, la coordinación es necesaria, ya que se ha demostrado que los retrasos en la inhalación reducen significativamente la entrega pulmonar ${ }^{36}$.

- Pueden utilizarse a cualquier edad, incluso en pacientes en asistencia respiratoria mecánica y con traqueostomía.

- No producen tos ni broncoespasmo reflejo, que a veces se presenta con el uso de pMDI sin espaciador.

Debido a que reducen el impacto en la orofaringe, los espaciadores y las cámaras de retención con válvula se recomiendan para el uso principalmente de corticosteroides. Sin embargo, aun así, es importante enjuagar la boca del paciente después de inhalar un corticosteroide con cualquiera de estos dispositivos, para retirar las partículas impactadas en la orofaring $\mathrm{e}^{10}$. 
Tabla 1. Medicamentos disponibles en México en dispositivo pMDI, DPI y niebla fina

\begin{tabular}{|c|c|c|c|}
\hline & Tipo de medicamento & Nombre genérico & Marcas \\
\hline \multirow[t]{9}{*}{ pMDI } & \multirow[t]{2}{*}{ Broncodilatadores de corta acción } & Salbutamol & $\begin{array}{l}\text { Ventolin }^{\circledR}, \text { Assal }^{\circledR}, \text { Aurosal }^{\circledR}, \text { Farmarest }^{\circledR} \text {, } \\
\text { Inbumed }^{\circledR}, \text { Salamol }^{\circledR} \text {, Salbutalan }\end{array}$ \\
\hline & & Bromuro de ipratropio & Atopyo ${ }^{\circledR}$ y Atrovent ${ }^{\circledR}$ \\
\hline & \multirow[t]{4}{*}{ Esteroides inhalados } & Beclometasona & $\begin{array}{l}\text { Ovar }{ }^{\circledR}, \text { Baclonet }^{\circledR}, \text { Beclazone }^{\circledR}, \\
\text { Dobipro }\end{array}$ \\
\hline & & Ciclesonida & Alvesco ${ }^{\circledR}$ \\
\hline & & Fluticasona & Flixotide ${ }^{\circledR}$ \\
\hline & & Budesonida & Numark $^{\circledR}$, Aerosial $^{\circledR}$, Jagodi ${ }^{\circledR}$ \\
\hline & \multirow[t]{3}{*}{$\begin{array}{l}\text { Combinación de esteroide inhalado } \\
\text { y broncodilatadores de larga acción }\end{array}$} & Fluticasona con salmeterol & Seretide Evohaler ${ }^{\circledR}$, Lasfligen $^{\circledR}$ \\
\hline & & Budesonida con formoterol & Vannair $^{\circledR}$ \\
\hline & & Mometasona con formoterol & Zenhale $^{\circledR}$ \\
\hline \multirow[t]{10}{*}{ DPI } & \multirow[t]{3}{*}{ Esteroides inhalados } & \multirow[t]{2}{*}{ Budesonida } & Dosis única: Miflonide Aerolizer ${ }^{\circledR}$ \\
\hline & & & Multidosis: Pulmicort Turbuhaler ${ }^{\circledR}$ \\
\hline & & Mometasona & Multidosis: Elovent Twisthaler ${ }^{\circledR}$ \\
\hline & \multirow{6}{*}{$\begin{array}{l}\text { Combinación de esteroide inhalado } \\
\text { y broncodilatadores de larga acción }\end{array}$} & \multirow[t]{2}{*}{ Fluticasona con salmeterol } & Dosis única: Nimbus y Ulfhinlas ${ }^{\circledR}$ \\
\hline & & & $\begin{array}{l}\text { Multidosis: Seretide Diskus e Irflosol } \\
\text { Forspiro }^{\circledR}\end{array}$ \\
\hline & & Budesonida con formoterol & Multidosis: Symbicort Turbuhaler ${ }^{\circledR}$ \\
\hline & & Bromuro de aclidinio con formoterol & Dosis única: Duaklir Genuair ${ }^{\circledR}$ \\
\hline & & Beclometasona con formoterol & Multidosis: Innovair Nexthaler ${ }^{\circledR}$ \\
\hline & & $\begin{array}{l}\text { Trifenatato de vilanterol con } \\
\text { fluticasona }\end{array}$ & Multidosis: Relvar Ellipta ${ }^{\circledR}$ \\
\hline & Broncodilatadores de larga acción & Fumarato de formoterol & Multidosis: 0xis Turbuhaler ${ }^{\circledR}$ \\
\hline \multirow[t]{2}{*}{ Niebla fina } & Broncodilatador de corta acción & $\begin{array}{l}\text { Salbutamol con bromuro de } \\
\text { ipratropio }\end{array}$ & Combivent Respimat ${ }^{\circledR}$ \\
\hline & Broncodilatador de larga acción & Tiotropio & Spiriva Respimat ${ }^{\circledR}$ \\
\hline
\end{tabular}

DPI: inhalador de polvo seco; pMDI: inhalador presurizado de dosis medida.

La mayoría de los espaciadores están hechos de materiales plásticos no conductores: la aerosolización y el manejo cotidiano inducen una carga electrostática en la superficie interna y externa de los mismos. El efecto neto de estas cargas electrostáticas es la atracción de partículas de aerosol hacia las paredes y la reducción significativa de la dosis disponible de aerosol para inhalación ${ }^{37}$. Se han desarrollado algunos espaciadores «sin estática», que se basan en el tipo de material plástico utilizado o en la presencia de una capa extremadamente delgada de metal en su superficie interna que reduce la estática. En las cámaras de retención con este tipo de materiales, el aerosol se mantiene suspendido por periodos más prolongados ${ }^{38}$.

Una recomendación para disminuir la estática en los dispositivos es que, tanto los nuevos como los previamente utilizados, se laven con detergente para trastes líquido diluido, no se enjuaguen y se dejen secar al aire libre. Estas medidas permitirán que se acumule un revestimiento con este material dentro del espaciador, que disminuirá la estática ${ }^{37,39}$, lo que incrementará la entrega de medicamento por el espaciador. El efecto del revestimiento con detergente sobre la carga electrostática disminuye después de una semana ${ }^{40}$, pero 


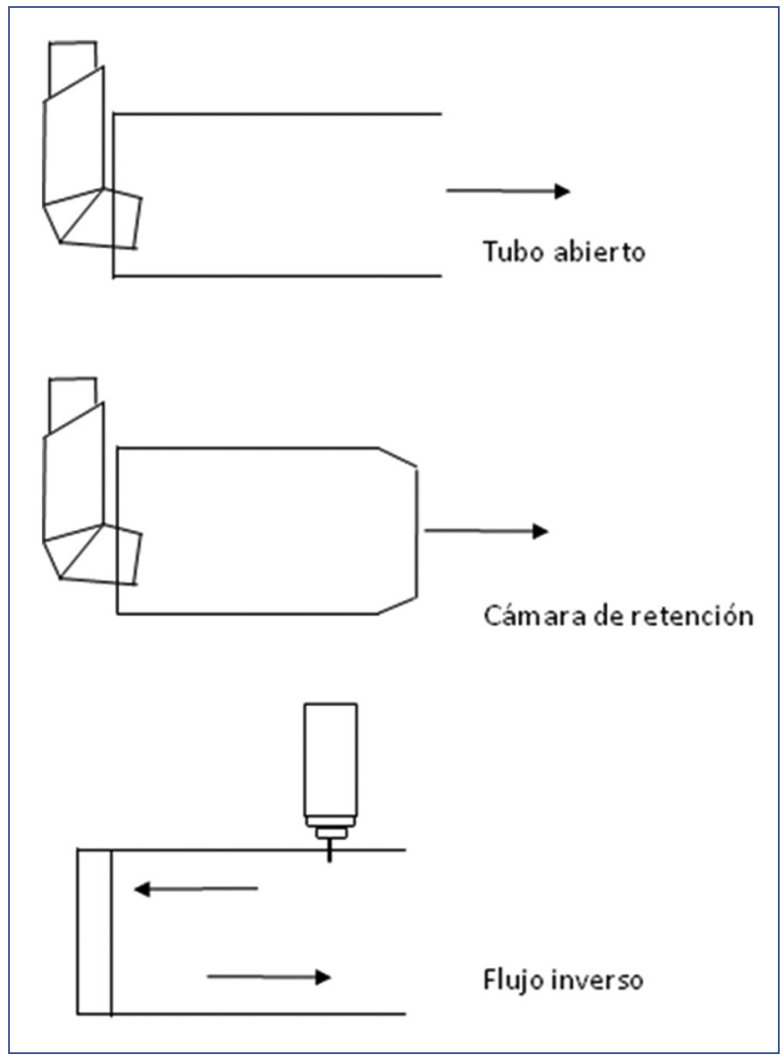

Figura 4. Tipos de dispositivos complementarios (cámara espaciadora) para inhaladores presurizados de dosis medida (modificado de Adkinson Jr. et al. ${ }^{29}$ ).

la eficiencia del depósito del fármaco a nivel pulmonar se sigue manteniendo durante cuatro semanas, por lo que se recomienda repetir el lavado en este tiempo ${ }^{41}$.

La principal desventaja de este tipo de dispositivos es que generalmente son voluminosos y menos portátiles que un pMDI.

\section{INHALADORES DE DOSIS MEDIDA PRESURIZADOS ACTIVADOS POR INHALACIÓN}

Se desarrollaron para superar el problema de mala coordinación de activación-inhalación con los pMDI estándar. Los inhaladores de dosis medida presurizados activados por inhalación (BA-pMDI) actualmente disponibles son el Autohaler ${ }^{\circledR}$, que automáticamente se acciona a velocidades de flujo inspiratorio de $\approx 30 \mathrm{l} / \mathrm{min}$, y el Easi-Breathe ${ }^{\circledR}$, que se activa a $20 \mathrm{l} / \mathrm{min}^{10}$.

\section{Inhaladores de polvo seco}

A principios de los setenta aparecieron los primeros DPI en los EE. UU. ${ }^{42}$, que se presentaron como una opción más amigable con el medio ambiente (al no contener productos fluorados), con mayor estabilidad química que las formulaciones líquidas ${ }^{28}$ y que requerían mínima coordinación entre la respiración y la activación del dispositivo ${ }^{43}$. El primero en aparecer fue el Spinhaler ${ }^{\circledR}$, dispositivo unidosis que perforaba una cápsula que se depositaba manualmente en una ranura y era inhalado por una pieza bucal. Conforme pasaron los años, se agregaron mejoras al diseño, dispositivos multidosis y sustancias acarreadoras que permitían una mejor entrega a nivel pulmonar.

Actualmente existen dos tipos básicos de DPI, según en el número de dosis que el dispositivo puede llevar: unidosis, donde el medicamento es suministrado en cápsulas individuales que se depositan manualmente por el paciente, y multidosis, que utilizan dosis medidas y selladas de fábrica, empacadas de tal manera que el dispositivo no tenga que ser recargado. De estos últimos, existen aquellos que tienen la formulación a granel (en masa) en un reservorio y que tienen un mecanismo incorporado para medir dosis individuales tras la activación, y aquellos con cantidades predosificadas y dispensadas de fábrica dentro de discos, cartuchos 0 tiras empacadas en blísteres ${ }^{10}$.

Desde el punto de vista del diseño, las partes primarias de un DPI son las mismas para todos los tipos de dispositivos. Consisten en una formulación en polvo, un mecanismo de dosificación que contiene (o mide) una sola dosis de medicamento, un principio de desaglomeración del polvo, que dispersa el polvo en la corriente de aire inhalado, y una pieza bucal ${ }^{44}$ (Fig. 3).

Los DPI disponibles actualmente se clasifican como dispositivos pasivos, porque utilizan la energía de la vibración que ejerce el paciente al inhalar sobre la pieza bucal para facilitar la desaglomeración del medicamento ${ }^{6} y$, por lo tanto, la entrega de la dosis depende del esfuerzo inspiratorio del paciente. Para cada inhalador hay un mínimo de energía, es decir, el flujo inspiratorio requerido para proporcionar una eficiente desagregación de la formulación, debajo del umbral mínimo de energía, el paciente no tendrá el efecto terapéutico del medicamento. La mayoría de los DPI comercialmente disponibles requieren de una tasa de flujo inspiratorio pico entre 30 y $60 \mathrm{l} / \mathrm{min}$ para la entrega del polvo, con algunas variaciones en los diferentes dispositivos. Es importante tener en cuenta que niños, adultos mayores y aquellos que experimenten una exacerbación grave pueden no ser capaces de generar el flujo inspiratorio suficiente para su adecuado funcionamiento, por lo que en estos pacientes se requerirá el uso de un dispositivo de inhalación diferente. 
Tabla 2. Comparación de distintos tipos de inhaladores

\begin{tabular}{|c|c|c|c|c|}
\hline Tipo y marca & $\begin{array}{l}\text { Tamaño de las } \\
\text { partículas }(\boldsymbol{p m})\end{array}$ & $\begin{array}{c}\text { Depósito pulmonar } \\
(\%)\end{array}$ & $\begin{array}{c}\text { Depósito faríngeo } \\
(\%)\end{array}$ & $\begin{array}{l}\text { Flujo inspiratorio } \\
\text { requerido (litros/min) }\end{array}$ \\
\hline $\begin{array}{l}\text { pMDI } \\
\text { General } \\
\text { ICP + Cámara } \\
\text { Partícula fina } \\
\text { Innovair } \\
\text { Alvesco }{ }^{\circledR}\end{array}$ & $\begin{array}{c}1.4-8 \\
2-3.2 \\
1-2 \\
1\end{array}$ & $\begin{array}{c}7.8-34 \\
11.2-68.3 \\
31-34 \\
50-52\end{array}$ & $\begin{array}{c}53.9-82.2 \\
31.2 \\
33-58 \\
32.9\end{array}$ & $\begin{array}{l}20-30 \\
20-30 \\
20-30 \\
20-30\end{array}$ \\
\hline $\begin{array}{l}\text { DPI } \\
\text { General } \\
\text { Monodosis } \\
\text { Aerolizer }^{\circledR} \\
\text { Breezhaler }^{\circledR} \\
\text { Handihaler }^{\circledR}\end{array}$ & $\begin{array}{c}1-2 \\
1.9-7.9 \\
2.8 \\
3.9\end{array}$ & $\begin{array}{c}25-30 \\
13-20 \\
39 \\
17.8\end{array}$ & $\begin{array}{c}70-75 \\
73 \\
45 \\
71\end{array}$ & $\begin{array}{l}30-60 \\
>90 \\
>90 \\
<50\end{array}$ \\
\hline $\begin{array}{l}\text { Multidosis } \\
\text { Diskus }^{\circledR} \\
\text { Easyhaler }^{\circledR} \\
\text { Ellipta }^{\circledR} \\
\text { Genuair }^{\circledR} \\
\text { Nexthaler }^{\circledR} \\
\text { Spiromax }^{\circledR} \\
\text { Turbuhaler }^{\circledR} \\
\text { Twisthaler }^{\circledR}\end{array}$ & $\begin{array}{c}3.5 \\
\overline{-} \\
2-4 \\
\overline{1.4-1.5} \\
\overline{-} \\
1.7-5.4 \\
2-2.2\end{array}$ & $\begin{array}{c}7.6 \\
18.5-31 \\
15-27 \\
30.1 \\
56 \\
28-49 \\
14.2-38 \\
36-37\end{array}$ & $\begin{array}{c}- \\
- \\
- \\
54.7 \\
43 \\
- \\
53-71.6 \\
-\end{array}$ & $\begin{array}{c}30-60 \\
<50 \\
<50 \\
60-90 \\
60-90 \\
60-90 \\
50-60 \\
<50\end{array}$ \\
\hline $\begin{array}{l}\text { Niebla fina } \\
\text { Respimat }^{\circledR}\end{array}$ & $1-2$ & $40-53$ & $19.3-39$ & $20-30$ \\
\hline
\end{tabular}

DPI: inhalador de polvo seco; ICP: inhalador de cartucho presurizado; pMDI: inhalador presurizado de dosis medida. Modificado de De Ávila, et al. ${ }^{49}$.

En general, los DPI actuales tienen una media de depósito en las vías respiratorias entre el 40 y el 80\% de la dosis medida ${ }^{45}$ (con una adecuada técnica de inhalación), que resulta similar e incluso ligeramente mejor que aquellos observados para los $\mathrm{pMDI}^{29}$. La mayoría de estos dispositivos se valen del uso de una sustancia acarreadora (lactosa o glucosa) que se mezcla con el medicamento y permite que el polvo pueda pasar más fácilmente fuera del dispositivo. La mayor parte de estas partículas, debido a su tamaño (20-65 $\mu \mathrm{m}$ ), se depositan en la orofaringe (por lo que se debe recomendar siempre el enjuague bucal), lo que permite que las partículas más pequeñas del medicamento $(1-2 \mu \mathrm{m})$ se entreguen a nivel pulmonar ${ }^{29}$.

Un punto a considerar es que la humedad del ambiente puede afectar marcadamente el medicamento suministrado desde el DPI. La dosis entregada a nivel pulmonar disminuye en un ambiente húmedo, probablemente como resultado de la aglutinación del polvo por la humedad añadida, por lo que es de suma importancia almacenar los DPI en un ambiente frío y seco. Otra desventaja es que estos dispositivos no pueden utilizarse en pacientes con asistencia respiratoria mecánica ni con traqueostomía.
En la actualidad, existen diferentes dispositivos DPI: Turbuhaler $^{\circledR}$, Diskus $^{\circledR}$, Eklira $^{\circledR}$, Irflosol $^{\circledR}$, Twisthaler $^{\circledR}$, Ellipta $^{\circledR}$, Diskhaler ${ }^{\circledR}$. En general, todos ellos funcionan bajo el mismo principio y cambian solo en algunas especificaciones, como el flujo inspiratorio, el uso de transportadores y el diseño (Tabla 1$)^{46}$.

\section{Inhalador de niebla fina}

La idea de desarrollar este tipo de dispositivos era combinar las ventajas de los pMDI y los nebulizadores. Son pequeños inhaladores portátiles y manuales, que no requieren un suplemento de energía (como los pMDI) para liberar el medicamento. Se presentan libres de propelente, en forma de una suave niebla, lo que disminuye la probabilidad de depósito orofaríngeo.

Actualmente se cuenta con el dispositivo conocido como Respimat ${ }^{\circledR}$ (Boehringer-Ingelheim). Este inhalador está disponible en tres presentaciones: bromuro de ipratotropio/salbutamol (Combivent ${ }^{\circledR}$ ), bromuro de tiotropio (Spiriva ${ }^{\circledR}$ ) y olodaterol (Striverdi ${ }^{\circledR}$ ).

La solución del medicamento es almacenada en una bolsa plegable dentro del cartucho del dispositivo. Respimat ${ }^{\circledR}$ tiene un resorte de tensión que, al ser torcido 
Tabla 3. Ventajas y desventajas de los diferentes tipos de dispositivos

\begin{tabular}{|c|c|c|}
\hline Tipo & Ventajas & Desventajas \\
\hline pMDI & $\begin{array}{l}\text { - Portátil y compacto } \\
\text { - Difícil de contaminar } \\
\text { - Menos costoso que otros inhaladores } \\
\text { - Dosis entregada y tamaño de la partícula } \\
\text { independiente de la maniobra de inhalación } \\
\text { - Adecuado para situaciones de urgencia } \\
\text { - Tiempo de tratamiento corto } \\
\text { - Disponible para la mayoría de las formulaciones } \\
\text { de medicamentos }\end{array}$ & $\begin{array}{l}\text { - Inhalación lenta y profunda (30 l/min) seguida de } \\
10 \text { s de sostener la respiración o } \\
\text { - Respiración lenta a volumen corriente empezando } \\
\text { inmediatamente después de la activación } \\
\text { - Activación de solo una dosis por inhalación }\end{array}$ \\
\hline pMDI con espaciador & $\begin{array}{l}\text { - Adecuado para niños pequeños y adultos mayores } \\
\text { - Reducción del depósito orofaríngeo } \\
\text { - Adecuado para situaciones de urgencia }\end{array}$ & $\begin{array}{l}\text { - Retraso, múltiples disparos y carga estática } \\
\text { reducen la biodisponibilidad pulmonar } \\
\text { - Voluminoso: mucho menos portable que el pMDI } \\
\text { solo } \\
\text { - La mayoría de los espaciadores están } \\
\text { específicamente diseñados para un pMDI }\end{array}$ \\
\hline DPI & $\begin{array}{l}\text { - Pequeño y portátil } \\
\text { - Activado por respiración } \\
\text { - Se requiere menor coordinación del paciente } \\
\text { - Poco tiempo de tratamiento } \\
\text { - Disponible para la mayoría de las sustancias }\end{array}$ & $\begin{array}{l}\text { - Se requiere de flujo inspiratorio moderado a alto } \\
\text { - No adecuado para niños }<4 \text { años } \\
\text { - Puede no ser apropiado para situaciones de } \\
\text { urgencia } \\
\text { - Algunos sensibles a la humedad } \\
\text { - No adecuado para la entrega de grandes dosis de } \\
\text { broncodilatadores }\end{array}$ \\
\hline
\end{tabular}

DPI: inhalador de polvo seco; pMDI: inhalador presurizado de dosis medida. Modificado de Broeders ME, et al. ${ }^{50}$.

a $180^{\circ}$, fuerza un volumen medido de solución de medicamento a través de un tubo capilar dentro de una microbomba; una vez que el paciente presiona el botón de liberación de dosis, la energía liberada del resorte impulsa la dosis dentro del uniblock, la parte estructural única de este dispositivo. El uniblock permite que la solución de medicamento pase a través de canales de filtro muy finos, y libera dos corrientes de chorro de solución de medicamento que convergen, y crea una fracción grande de gotitas respirables con baja velocidad (Fig. 3) 47. $^{4}$.

El inhalador de niebla fina aerosoliza partículas entre 1 a $5.8 \mu \mathrm{m}$, lo que ayuda a evitar la pérdida del medicamento durante la exhalación y a facilitar el depósito pulmonar a través del mecanismo de sedimentación ${ }^{48}$, con un depósito pulmonar de alrededor del 40 al 53\%.

\section{Selección del inhalador}

Existen factores importantes cuando se selecciona un inhalador ${ }^{49}$ (Tabla 2). Por ejemplo, el tamaño de la partícula, la preferencia del paciente, la habilidad para utilizar y generar un nivel apropiado de flujo inspiratorio para el dispositivo ${ }^{50,51}$, y las ventajas y desventajas descritas para cada uno de ellos (Tabla 3). En población pediátrica existen problemas específicos que hay que tomar en cuenta cuando se prescribe un inhalador; por ejemplo, se sabe que el llanto reduce de manera importante la entrega de aerosol a las vías aéreas periféricas, por lo que se deberá recomendar un ambiente tranquilo que incremente la comodidad de este grupo de pacientes durante la administración de los medicamentos inhalados.

A pesar de no estar incluido en esta revisión, un nebulizador puede ser utilizado como una alternativa adecuada, especialmente en el escenario de síntomas agudos; sin embargo, los nebulizadores no se recomiendan para el tratamiento crónico y continuo porque son más caros, la administración del medicamento consume más tiempo y requiere de mayor mantenimiento. Además, los nebulizadores son fuentes potenciales de contaminación microbiana del tracto respiratorio: tanto los nebulizadores portátiles en jet como los ultrasónicos se han asociado con neumonía nosocomial ${ }^{52}$.

\section{Técnica de inhalación}

Independientemente del dispositivo que se recomiende, es muy importante la capacitación del paciente sobre el adecuado uso (Tablas 4, 5 y 6). Existen varios videos informativos disponibles en Internet ${ }^{53-55}$. 
Tabla 4. Técnica para uso de pMDI

1. Quite la tapa de la pieza bucal del inhalador

2. Agite el inhalador (no es necesario para algunos pMDI con HFA. El paciente debe revisar el folleto de instrucciones)

3. Sostenga el inhalador en posición vertical

4. Exhale todo el aire

5. Coloque la pieza bucal del inhalador entre sus labios y dientes o coloque la pieza bucal a $4 \mathrm{~cm}$ de distancia de su boca; mantenga su lengua abajo y fuera del camino de la pieza bucal

6. Dispare el inhalador justo después de una inhalación muy lenta y muy profunda

7. Continúe inhalando muy lentamente hasta que sus pulmones se llenen (debe tomar por lo menos 5 segundos en llenar sus pulmones)

8. Retire el inhalador de su boca y aguante la respiración mientras cuenta hasta 10 o lo más que pueda

9. Exhale lentamente

HFA: hidrofluoroalcano; pMDI: inhalador presurizado de dosis medida. Modificado de Sanchis J, et al. ${ }^{53}$.

Tabla 5. Técnica para uso de pMDI con espaciador o cámara espaciadora

1. Quite la tapa de la pieza bucal

2. Active el inhalador si es la primera vez que se usa, si ha estado varios días sin uso, o si se cayó. El activar el inhalador implica agitarlo y dispararlo al aire (lejos de la cara) hasta cuatro ocasiones (revise las instrucciones del dispositivo)

3. Inserte el pMDI en el espaciador

4. Agite el pMDI durante 5 segundos de forma vigorosa

5. Mantenga el inhalador en posición vertical entre su dedo índice y el pulgar, puede usar la otra mano para sujetar el espaciador

6. Exhale de forma normal a través de su boca

7. Coloque la pieza bucal entre sus dientes y cierre los labios firmemente alrededor de la pieza bucal del espaciador (si usa una máscara facial, colóquela sobre la nariz y la boca)

8. Asegúrese de que su lengua no bloquee la pieza bucal del espaciador

9. Presione el contenedor del pMDI con su dedo índice para que salga el medicamento

10. Al mismo tiempo, inhale profunda y lentamente a través de su boca hasta que los pulmones se llenen por completo; esto le llevará de 3 a 5 segundos

11. Mantenga la medicina durante 5 a 10 segundos. Si no puede inspirar de forma completa o no puede detener la respiración el tiempo suficiente, puede inhalar una segunda ocasión para vaciar el espaciador y detener el aire de nuevo por otros 5 segundos

12. Si usted requiere más de un disparo, espere de 15 a 30 segundos entre disparo y disparo. Agite de nuevo el pMDI antes del siguiente disparo. No dé dos disparos en el espaciador y trate de vaciarlo con una inhalación

13. Cuando finalice, tape la pieza bucal

14. Si su medicamento contiene un esteroide (a veces llamado glucocorticoide o corticoesteroide), enjuague su boca y haga colutorios con agua después de su uso. Escupa el agua, no la trague

15. Puede usar su espaciador para más de un medicamento; quite el primero para usar los siguientes

pMDI: inhalador presurizado de dosis medida.

A pesar de la terapia adecuada, cuando se evalúa a un paciente con síntomas de asma no controlado, una de las preguntas que deben hacerse es si el paciente está utilizando la medicación prescrita. Las tasas de adherencia al medicamento en pacientes asmáticos son bajas. Esto se debe al mal control de la enfermedad, exacerbaciones, alto costo del tratamiento y falta de acceso a los servicios de salud. 
Tabla 6. Técnica de para uso de DPI Turbuhaler ${ }^{\circledR}$ y Diskus ${ }^{\circledR}$

\section{Técnica de Turbuhaler}

1. Retire la tapa del inhalador

2. Mantenga el inhalador en posición vertical

3. Gire el mango en sentido contrario a las manecillas del reloj y luego de regreso hasta escuchar un click

4. Exhale a volumen residual

5. Exhale lejos de la pieza bucal

6. Coloque la boquilla entre los dientes y los labios

7. Inhale con fuerza y profundamente

8. Sostenga la respiración durante 5 segundos

9. Exhale lejos de la pieza bucal (coloque la tapa después del uso del Turbuhaler)
Técnica de Diskus

1. Abra el inhalador

2. Empuje la palanca hacia atrás por completo

3. Exhale a volumen residual

4. Exhale lejos de la pieza bucal

5. Coloque la boquilla entre los dientes y los labios

6. Inhale con fuerza y profundamente

7. Sostenga la respiración durante 5 segundos

8. Exhale lejos de la pieza bucal

9. Cierre el inhalador

DPI: inhalador de polvo seco.

Modificado de Basheti IA, et al..$^{55}$.

Es frecuente que los pacientes utilicen sus inhaladores de manera incorrecta. En algunos casos, solo del 46 al $59 \%$ de los pacientes realizan una técnica de inhalación eficiente ${ }^{56}$, e incluso el $25 \%$ nunca han recibido capacitación con una técnica del correcto uso de inhalador ${ }^{57}$. Dos revisiones sistemáticas sobre la problemática del uso de inhaladores indican que solamente el $31 \%$ de asmáticos que utilizan espaciadores lo hacen con la técnica adecuada. Los errores más frecuentes son la mala coordinación entre el disparo y la inhalación y el no poder retener la respiración después de la deposición del medicamento, lo que llega a ocurrir hasta en el $45 \%$ de los $\operatorname{casos}^{58}$.

Entre los profesionales de la salud, la técnica fue correcta en solamente el $15.5 \%$, cifra que ha disminuido en los últimos años ${ }^{59}$.

A la fecha no existen una serie de pasos universales para todos los dispositivos; sin embargo, cada uno cuenta con unas instrucciones de uso muy similares.

Las demostraciones personales o en grupos pequeños son las más eficaces, y es importante no perder de vista que el reentrenamiento periódico es siempre necesario ${ }^{60}$. La técnica de inhalación mejora a casi el doble (del $57.4 \%$ al $97.9 \%$ ) después de tres sesiones de entrenamiento ${ }^{61}$.

En conclusión, el control óptimo del asma depende, en gran medida de la terapéutica seleccionada, del dispositivo empleado y de la eliminación de factores que reducen la adherencia del paciente al tratamiento. La selección del dispositivo siempre deberá hacerse de forma conjunta con el paciente, evaluando los pros y los contras de cada uno de ellos.

\section{Responsabilidades éticas}

Protección de personas y animales. Los autores declaran que para esta investigación no se han realizado experimentos en seres humanos ni en animales.

Confidencialidad de los datos. Los autores declaran que han seguido los protocolos de su centro de trabajo sobre la publicación de datos de pacientes.

Derecho a la privacidad y consentimiento informado. Los autores declaran que en este artículo no aparecen datos de pacientes.

\section{Conflicto de intereses}

Los autores declaran no tener ningún conflicto de intereses.

\section{Financiamiento}

No se recibió ningún tipo de financiamiento para la realización de la presente revisión.

\section{Bibliografía}

1. Pawankar R, Canonica GW, Holgate ST, Lockey RF, editores. The WAO white book on allergy. United Kingdom: World Allergy Organization; 2011.

2. Worldwide variation in prevalence of symptoms of asthma, allergic rhinoconjunctivitis, and atopic eczema: ISAAC. The International Study of Asthma and Allergies in Childhood (ISAAC) Steering Committee. Lancet. 1998;351:1225-32. 
3. Dolovich MB, Dhand R. Aerosol drug delivery: developments in device design and clinical use. Lancet. 2011;377:1032-45.

4. Rottier BL, Rubin BK. Asthma medication delivery: mists and myths. Paediatr Respir Rev. 2013;14:112-8.

5. Bjermer L. The importance of continuity in inhaler device choice for asthma and chronic obstructive pulmonary disease. Respiration. 2014:88:346-52.

6. Stein SW, Thiel CG. The history of therapeutic aerosols: a chronological review. J Aerosol Med Pulm Drug Deliv. 2017;30:20-41.

7. Sanders M. Inhalation therapy: an historical review. Prim Care Respir J. 2007:16:71-81.

8. Thiel CG. From Susie's question to CFC-free: an inventor's perspective on forty years of MDI development and regulation. Respir Drug Deliv. 1996:1:115-23.

9. Nikander K, Nicholls C, Denyer J, Pritchard J. The evolution of spacers and valved holding chambers. J Aerosol Med Pulm Drug Deliv. 2014;27:S4-23.

10. Mitchell JP. What the pulmonary specialist should know about the new inhalation therapies. Eur Respir J. 2012;39:1054-5.

11. Laube BL, Janssens HM, de Jongh FH, Devadason SG, Dhand R, Diot $P$, et al. What the pulmonary specialist should know about the new inhalation therapies. Eur Respir J. 2011;39:1308-31.

12. Rottier BL, Rubin BK. Asthma medication delivery: mists and myths. Paediatr Respir Rev. 2013;14:112-8; quiz 118, 137-8.

13. Labiris NR, Dolovich MB. Pulmonary drug delivery. Part I: physiological factors affecting therapeutic effective Ness of aeroolized medicatios. $\mathrm{Br}$ $\mathrm{J}$ Clin Pharmacol. 2003;56:588-99.

14. Muchão FP, Filho LV. Advances in inhalation therapy in pediatrics. J Pediatr (Rio J). 2010;86:367-76.

15. Haughney J, Price D, Barnes NC, Virchow JC, Roche N, Chrystyn H. Choosing inhaler devices for people with asthma: current knowledge and outstanding research needs. Respir Med. 2010;104:1237-45.

16. Self TH, Pinner NA, Sowell RS, Headley AS. Does it really matter what volume to exhale before using asthma inhalation devices? J Asthma. 2009:46:212-6.

17. Cheng YS. Mechanisms of pharmaceutical aerosol deposition in the respiratory tract. AAPS PharmSciTech. 2014;15:630-40.

18. Myers TR. The science guiding selection of an aerosol delivery device. Respir Care. 2013:58:1963-73.

19. Dal-Negro RW. Dry powder inhalers and the right things to remember: a concept review. Multidiscip Respir Med. 2015;10:13.

20. Dolovich MB, Ahrens RC, Hess DR, Anderson P, Dhand R, Rau JL; American College of Chest Physicians, American College of Asthma, Allergy and Immunology. Device selection and outcomes of aerosol therapy: evidence-guidelines. Chest. 2005;127:335-71.

21. Amirav I, Newhouse MT, Mansour Y. Measurement of peak inspiratory flow with in-check dial device to simulate low-resistance (Diskus) and high resistance (Turbohaler) dry powder inhalers in children with asthma. Pediatr Pulmonol. 2005:39:447-51.

22. Smaldone GC. Drug delivery via aerosol systems: concept of "aerosol inhaled". J Aerosol Med. 1991;4:229-35.

23. Hendeles L, Colice GL, Meyer RJ. Withdrawal of albuterol inhalers containing chlorofluorocarbon propellants. N Engl J Med. 2007:356:1344-51.

24. Vaswani SK, Creticos PS. Metered dose inhaler: past, present, and future. Ann Allergy Asthma Immunol. 1998;80:11-9.

25. Cummings RH. Pressurized metered dose inhalers: chlorofluorocarbon to hydrofluoroalkane transit ion-valve performance. J Allergy Clin Immunol. 1999;104:S230-6.

26. Newman SP. Principles of metered-dose inhaler design. Respir Care. 2005:50:1177-90.

27. Azouz W, Chrystyn H. Clarifyng the dilemas about inhalation techniques for dry poder inhalers: integrating science with clinical practice. Prim Care Respir J. 2012;21:208-13.

28. Ibrahim M, Verma R, Garcia-Contreras L. Inhalation drug delivery devices: techonology update. Med Devices (Auckl). 2015;8:131-9.

29. Adkinson Jr. NF, Bochner BS, Burks AS, Busse WW, Holgate ST, Lemanske Jr. RF, et al. Middleton's allergy: principles and practice. Maryland: Elsevier; 2008. p. 1-1924.

30. Newman SP, Newhouse MT. Effect of add-on devices for aerosol drug delivery: deposition studies and clinical aspects. J Aerosol Med. 1996:9:55-70.

31. Nair A, Menzies D, Hopkins P, McFarlane L, Lipworth BJ. In vivo comparison of the relative systemic bioavailability of fluticasone propionate from three antistatic spacers and a metered dose inhaler. $\mathrm{Br} \mathrm{J}$ Clin Pharmacol. 2009:67:191-8

32. Nair A, Menzies D, Barnes M, Burns P, McFarlane L, Lipworth BJ. Respirable dose delivery of fluticasone propionate from a small valved holding chamber, a compact breath actuated integrated vortex device and a metered dose inhaler. Br J Clin Pharmacol. 2008;66:20-6.

33. Liu CL, Lu YT. Bronchodilatation effects of a small volume spacer used with a metered-dose inhaler. J Asthma. 2009:46:637-41.
34. Berger WE, Bensch GW, Weinstein SF, Skoner DP, Prenner BM, Shekar $\mathrm{T}$, et al. Bronchodilation with mometasone furoate/formoterol fumarate administered by metered-dose inhaler with and without a spacer in children with persistent asthma. Pediatr Pulmonol. 2014:49:441-50.

35. Dolovich M. Lung dose, distribution, and clinical response to therapeutic aerosols. Aerosol Sci Tech. 1993;18:230-40.

36. Slator L, von Hollen D, Sandell D, Hatley RH. In vitro comparison of the effect of inhalation delay and flow rate on the emitted dose from three valved holding chambers. J Aerosol Med Pulm Drug Deliv. 2014; 27:S37-43.

37. Wildhaber JH, Waterer GW, Hall GL, Summers QA. Reducing electrostatic charge on spacer devices and bronchodilator response. $\mathrm{Br} \mathrm{J}$ Clin Pharmacol. 2000;50:277-80.

38. Rau JL, Coppolo DP, Nagel MW, Avvakoumova VI, Doyle CC Wiersema KJ, et al. The importance of nonelectrostatic materials in holding chambers for delivery of hydrofluoroalkane albuterol. Respir Care. 2006;51:503-10.

39. Anhoj J, Bisgaard H, Lipworth BJ. Effect of electrostatic charge in plastic spacers on the lung delivery of HFA-salbutamol in children. $\mathrm{Br} \mathrm{J}$ Clin Pharmacol. 1999;47:333-6.

40. Janssens HM, Heijnen EM, de Jong VM, Hop WC, Holland WP, de Jongste JC, et al. Aerosol delivery from spacers in wheezy infants: a daily life study. Eur Respir J. 2000;16:850-6.

41. Piérart F, Wildhaber JH, Vrancken I, Devadason SG, Le Souëf PN. Washing plastic spacers in household detergent reduces electrostatic charge and greatly improves delivery. Eur Respir J. 1999;13:673-8.

42. Bell JH, Hartley PS, Cox JSG. Dry powder aerosol I: a new powder inhalation device. J Pharm Sci. 1971:60:1559-64.

43. Atkins PJ. Dry powder inhalers: an overview. Respir Care. 2005; 50:1304-12.

44. Muralidharan P, Hayes D Jr, Mansour HM. Dry powder inhalers in COPD, lung inflammation and pulmonary infections. Expert Opin Drug Deliv. 2015; $12: 947-62$

45. Weers $\mathrm{J}$, Clark $\mathrm{A}$. The impact of inspiratory flow rate on drug delivery to the lungs with dry powder inhalers. Pharm Res. 2017:34:507-28.

46. Mehta P. Dry powder inhalers: a focus on advancements in novel drug delivery systems. J Drug Deliv. 2016;2016:8290963.

47. Dalby RN, Eicher J, Zierenberg B. Development of Respimat $₫$ Soft Mist ${ }^{\mathrm{T} M}$ inhaler and its clinical utility in respiratory disorders. Med Devices (Auckl). 2011;4:145-55

48. Dahl R, Kaplan A. A systematic review of comparative studies of tiotropium Respimat $\Omega$ and tiotropium HandiHaler $\Omega$ in patients with chronic obstructive pulmonary disease: does inhaler choice matter? CBMC Pulm Med. 2016;16:135

49. De Ávila Cabezón GR, González Rey J, Balaguer Balaguer EM. Las 4 reglas de la terapia inhalada. Galicia: Sogami; 2015

50. Broeders ME, Sanchis J, Levy ML, Crompton GK, Dekhuijzen PN; ADMIT Working Group. The ADMIT series-issues in inhalation therapy. 2. Improving technique and clinical effectiveness. Prim Care Respir J. 2009;18:76-82.

51. Pedersen S, Dubus JC, Crompton GK; ADMIT Working Group. The ADMIT series-issues in inhalation therapy. 5. Inhaler selection in children with asthma. Prim Care Respir J. 2010:19:209-16.

52. Ida Y, Ohnishi H, Araki K, Saito R, Kawai S, Watanabe T. Efficient management and maintenance of ultrasonic nebulizers to prevent microbial contamination. World J Methodol. 2016:6:126-32.

53. Sanchis J, Corrigan C, Levy ML, Viejo JL; ADMIT Group. Inhaler devices-from theory to practice. Respir Med. 2013;107:495-502.

54. Youtube.com [Internet]. Disponibles en: https://youtu.be/nSmf1rm1pnw; https://youtu.be/2n4eylibyFY; https://youtu.be/jKVrOyu_p94; https://youtu.be/ZaRxwcpyduk; https://youtu.be/bJAF_g4sC6I.

55. Basheti IA, Bosnic-Anticevich SZ, Armour CL, Reddel HK. Checklists for powder inhaler technique: a review and recommendations. Respir Care. 2014;59:1140-54

56. Cochrane MG, Bala MV, Downs KE, Mauskopf J, Ben-Joseph RH. Inhaled corticosteroids for asthma therapy: patient compliance, devices, and inhalation technique. Chest. 2000;117:542-50.

57. Molimard M, Raherison C, Lignot S, Depont F, Abouelfath A, Moore N. Assessment of handling of inhaler devices in real life: an observational study in 3811 patients in primary care. J Aerosol Med. 2003:16:249-54.

58. Sanchis J, Gich I, Pedersen S; Aerosol Drug Management Improvement Team (ADMIT). Systematic review of errors in inhaler use: has patient technique improved over time? Chest. 2016:150:394-406.

59. Plaza V, Giner J, Rodrigo GJ, Dolovich MB, Sanchis J. Errors in the use of inhalers by health care professionals: a systematic review. J Allergy Clin Immunol Pract. 2018;6:987-95.

60. Scichilone N. Asthma control: the right inhaler for the right patient. Adv Ther. 2015;32:285-92.

61. Sumino K, Cabana MD. Medication adherence in asthma patients. Curr Opin Pulm Med. 2013:19:49-53. 